\title{
Noninterference via Symbolic Execution ${ }^{\star}$
}

\author{
Dimiter Milushev, Wim Beck, and Dave Clarke \\ IBBT-DistriNet, KU Leuven, Heverlee, Belgium
}

\begin{abstract}
Noninterference is a high-level security property that guarantees the absence of illicit information flow at runtime. Noninterference can be enforced statically using information flow type systems; however, these are criticized for being overly conservative and rejecting secure programs. More precision can be achieved by using program logics, but such an approach lacks its own verification tools. In this work we propose a novel, alternative approach: utilizing symbolic execution in combination with ideas from program logics in an attempt to increase the precision of analyses and automate noninterference testing. Dealing with policies incorporating declassification is also explored. The feasibility of the proposal is illustrated using a prototype tool based on the KLEE symbolic execution engine.
\end{abstract}

Keywords: Noninterference, declassification, symbolic execution, testing.

\section{Introduction}

Noninterference is a high-level security property, prohibiting information leaks through the executions of a program. The typical program model for expressing noninterference assumes the following: public and secret inputs are given to a program; public and secret outputs are observable as a result of the program runs. In this context, noninterference is a policy stipulating that public outputs of a program should be functionally dependent on public inputs only, and not on secret inputs. The policy has been substantially studied in the languagebased security community 16 and typically relies on information flow type systems [15 21 22]; however, these are criticized for being conservative and rejecting many secure programs.

An alternative approach proposes the use of program logics for expressing noninterference. Such an approach was introduced by Darvas, Hähnle and Sands [8], who used dynamic logic to verify noninterference for sequential Java programs. One key observation they made is that noninterference (which is not a property and hence not directly expressible in program logics) on some program $P$ is reducible to a property on the sequential composition $P ; P^{\prime}$ of the program with itself. More precisely, noninterference can be characterized as the following

\footnotetext{
* This research is partly funded by the EU project FP7-231620 HATS: Highly Adaptable and Trustworthy Software using Formal Models (http://www.hats-project. $\mathrm{eu}$.
} 
quadruple: $\left\{\bar{l}=\overline{l^{\prime}}\right\} P ; P^{\prime}\left\{\bar{l}=\overline{l^{\prime}}\right\}$. Here, $P^{\prime}$ is the same program as $P$ with all variables renamed, $\bar{l}$ are the low variables of $P$ and $\overline{l^{\prime}}$ are the low variables of $P^{\prime}$. Barthe, Argenio and Rezk 4 based their characterization of noninterference in Hoare and temporal logics on similar ideas; they also coined the term self-composition for the construct $P ; P^{\prime}$. The program logics approaches provide more precision in specifications, but do not have their own verification tools; in addition, it is not clear how to reuse existing tools and techniques.

Terauchi and Aiken [20] note that self-composition is impractical. They point out that for the purpose of verification of noninterference, some nontrivial, partial-correctness condition that holds between $P$ and $P^{\prime}$ has to be found; and finding it is impractical. They also argue that in order to be useful for practical verification, self-composition needs to take into account the structure of a selfcomposed program and the resulting symmetry and redundancy. They propose a type-directed transformation for a simple imperative language to deal with the problems they identify.

Symbolic execution has already been used for verification of secure information flow [8]. The approach offers high precision but unfortunately requires considerable user interaction and verification expertise, needed for adding loop invariants, establishing induction hypotheses, instantiating and unwinding loops etc. Due to this fact, similar approaches are often criticized as being of limited practical significance for developers. In this work we attempt to remedy such limitations and propose the use of symbolic execution as a basis for a noninterference testing tool. This is advantageous because it is automatic and gives developers an efficient, practical way of testing for noninterference bugs. The proposed approach is essentially an under-approximation of the problem and this has two important consequences: on the positive side, it is automatic and precise; on the other hand, it usually cannot discover all bugs. Nevertheless, combined with the observation that typically most bugs are shallow, the approach gives developers a powerful tool, without requiring them to understand and write complex specifications.

More concretely, the advocated approach is based on utilizing symbolic execution in combination with a form of self-composition in an attempt to automate noninterference testing. We start off with Terauchi and Aiken's transformation and accommodate additional language features, such as dealing with procedures and dynamically allocated data structures. The approach essentially interleaves two copies of a program and then uses dynamic symbolic execution to try to extract all possible paths in the program. Conditions on two disjoint program stores are generated in order to express the desired security policy via assert statements. The resultant program is analyzed by the symbolic execution engine: if a bug is found, the program is not secure and the developer is informed about it; the proposed approach typically cannot guarantee that a program is secure. On secure programs, a tool based on this approach will often run indefinitely without producing any error message. On insecure programs it will typically run indefinitely but still discover security bugs and thus would indeed 
be useful. On concrete programs, our prototype tool has been able to discover instances of all the known patterns of insecurity we have found in the literature.

The contributions of the work are: first, a proposal to use symbolic execution in order to automatically specify and check a notion of plain noninterference and one incorporating declassification; second, an illustration of what is needed to transfer the ideas to a programming language having procedures and dynamic memory allocation (heap); finally, a prototype tool based on the KLEE symbolic execution tool [6] illustrating the feasibility of the approach. The rest of the work is structured as follows. Section 2 provides some background. Sections 3 presents the proposed approach. Section 4 presents our prototypical tool and experimental results. Finally, Sections 5 and 6 are left for the related work and conclusion.

\section{Background}

\subsection{Noninterference}

Intuitively, noninterference stipulates that public outputs of a program should be functionally dependent on public inputs only, and not on secret inputs. Define a store $\mathrm{m}$ to be a mapping from program variables from some set Var to values from set $\mathcal{V}$. The notation $\left.\mathrm{m}\right|_{X}$ is a restriction of the store to variables from domain $X ;(\mathrm{m}, P)$ denotes the final store after execution of program $P$ with initial store $\mathrm{m}$ and $(\mathrm{m}, P)=\perp$ signifies that the program diverges (does not terminate or terminates in an (unobservable) exceptional state). Finally, $\approx_{p}$ signifies the pointwise extension of equality to stores. The definition of termination insensitive information flow can be formulated as follows:

Definition 1. (Secure information flow [20]) A program $P$ with high security variables $H=\left\{h_{1}, \ldots, h_{i}\right\}$ and low security variables $L=\left\{l_{1}, \ldots, l_{j}\right\}$ is secure iff for all possible stores $m_{1}$ and $m_{2}$ such that $\left.\left.m_{1}\right|_{L} \approx_{p} m_{2}\right|_{L}$, we have that

$$
\left.\left.\left(\left(m_{1}, P\right) \neq \perp \wedge\left(m_{2}, P\right) \neq \perp\right) \Longrightarrow\left(m_{1}, P\right)\right|_{L} \approx_{p}\left(m_{2}, P\right)\right|_{L} .
$$

There is an obvious way to show that a program $P$ is not secure by Definition 1 . namely by finding two stores $\mathrm{m}_{i}$ and $\mathrm{m}_{j}$ such that $\left.\left.\mathrm{m}_{i}\right|_{L} \approx_{p} \mathrm{~m}_{j}\right|_{L},\left(\mathrm{~m}_{i}, P\right) \neq \perp \wedge$ $\left(\mathrm{m}_{j}, P\right) \neq \perp$, and $\left.\left.\left(\mathrm{m}_{i}, P\right)\right|_{L} \not_{p}\left(\mathrm{~m}_{j}, P\right)\right|_{L}$.

Program 1.1 also referred to as $P_{1}$, illustrates implicit information flow. Let $H=\{i, j\}, L=\{l\}$. Observing the value of variable $l$ discloses whether the average of the two secret values is greater than 1000 .

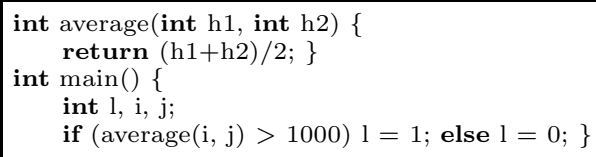

Program 1.1. Implicit information flow 
The implicit flow can be detected using Definition 1 Let $\mathrm{m}_{1}$ and $\mathrm{m}_{2}$ be such that $\mathrm{m}_{1}(i)=1000, \mathrm{~m}_{1}(j)=900, \mathrm{~m}_{1}(l)=0, \mathrm{~m}_{2}(i)=800 \mathrm{~m}_{2}(j)=1400$ and $\mathrm{m}_{2}(l)=0$. We have that $\left.\left.\mathrm{m}_{1}\right|_{L} \approx_{p} \mathrm{~m}_{2}\right|_{L},\left(\mathrm{~m}_{1}, P_{1}\right) \neq \perp \wedge\left(\mathrm{m}_{2}, P_{1}\right) \neq \perp$, but at the end of execution $\left(\mathrm{m}_{1}, P_{1}\right)(l)=0$ and $\left(\mathrm{m}_{2}, P_{2}\right)(l)=1$; thus $\left.\left.\left(\mathrm{m}_{1}, P_{1}\right)\right|_{L} \not \varpi_{p}\left(\mathrm{~m}_{2}, P_{1}\right)\right|_{L}$ implies that $P_{1}$ is insecure.

\subsection{Declassification}

Most useful computing systems have to release sensitive information as a part of their functionality (e.g. password checking, shopping for digital content, online games). Thus noninterference is often too strict for realistic systems; the usual solution is weakening the policy with declassification, a mechanism for releasing sensitive information. An important problem of declassification is to guarantee precisely what is being leaked and to ensure that the mechanism cannot be abused into leaking more [18.

More formally, consider program $P$ on stores $\mathrm{m}_{1}$ and $\mathrm{m}_{2}$. Recall that $\approx_{p}$ signifies the pointwise extension of equality to stores and $\left.\mathrm{m}\right|_{L}$ is a restriction of the store to variables from domain $L$. Let $\psi$ be the predicate defined as $\left.\left.\mathrm{m}_{1}\right|_{L} \approx_{p} \mathrm{~m}_{2}\right|_{L}$. Noninterference can be given as the following quadruple $\{\psi\}\left(\mathrm{m}_{1}, P\right) ;\left(\mathrm{m}_{2}, P\right)\{\psi\}$. If $\psi_{\text {decl }}$ is a predicate on high variables, specifying what is to be declassified by $P$, then noninterference with declassification can be expressed as follows: $\left\{\psi \wedge \psi_{\text {decl }}\right\}\left(\mathrm{m}_{1}, P\right) ;\left(\mathrm{m}_{2}, P\right)\{\psi\}[\underline{3}$.

For instance, in Program 1.1 the policy might be that it is admissible to reveal some fact about the average (i.e. whether (average $(i, j)>1000)$ holds) but not more than that. Then, in addition to the usual noninterference condition, $\psi_{\text {decl }}$ will be instantiated with $\left(\left(\operatorname{average}\left(i_{1}, j_{1}\right)>1000\right) \Leftrightarrow\left(\operatorname{average}\left(i_{2}, j_{2}\right)>1000\right)\right)$ (note that $i_{k}$ is shortcut for $\mathrm{m}_{k}(i)$ for $k \in\{1,2\}$ ).

Other dimensions of declassification are about who controls information release, where in the system does declassification occur and finally when can information be declassified [18. In this work, we focus on the what dimension.

\subsection{Symbolic Execution}

Symbolic execution [13] is a program analysis technique used to investigate the possible execution traces of a program. The idea is to replace program inputs with input symbols and thus instead of executing the program with concrete values, to execute it with symbolic expressions over the input symbols. When the program encounters a conditional branch statement, execution is forked because there are no concrete values to evaluate the condition: whether any or both of these branches are reachable is checked by a constraint solver. Loops can be seen as conditional statements encountered multiple times and they are lazily unrolled, possibly an infinite number of times. The conjunction of all conditions encountered on the branches of a single path is called a path condition.

Symbolic execution is a general approach that can be used to check or prove a range of properties of programs. Properties can be expressed using assert statements. 
Dynamic symbolic execution, also called concolic execution [19] or DART [1], is a variant of the technique interleaving concrete and symbolic execution. The idea is simple: first, gather the constraints for some path by monitoring program execution with some arbitrary, concrete inputs; then, systematically explore new execution paths by negating parts of the initial path condition. In this work we are going to use KLEE [6], an automatic symbolic execution tool built on top of the LLVM compiler infrastructure; the tool is used for both illustrations of the purposed approach and as a basis of our prototype tool.

\section{Approach}

\subsection{Overview}

The approach proposed in this paper starts with partitioning the program input variables into public and secret, and respectively annotating them. This is the only obligation on behalf of the developer, the rest is automatic. Then the variables are made symbolic and a type-directed transformation adopted from the work of Terauchi and Aiken [20] is applied to the program. The transformation is a variant of the self-compositional approach. It (the transformation) provides the method used for interleaving the candidate program with itself, which is needed to express noninterference as a property. We develop certain extensions of the transformation in order to deal with aspects of procedures and dynamically allocated data structures; the latter require reasoning about the heap and a modified definition of noninterference. After the transformation is complete assertions specifying the noninterference policy are placed. Then the symbolic execution tool is used as a program analysis tool for noninterference. If it is able to fully analyze all possible paths in the transformed program (paths have to be finite), a tool based on the approach can decide whether the program is secure or not. Otherwise the tool may either eventually return an error(s) or simply keep running without returning any error. In the latter cases the proposed approach is useful even if it cannot cover the whole state space, as it will still discover bugs in the covered part; moreover, the approach offers precision, i.e. lack of false-positives. Because of the nature of typical bugs (being shallow), this strategy often turns out to be very helpful.

\subsection{Transformation of a Basic Language}

We start off by illustrating how to transform a program for a minimal language including variable declarations and assignments, while loops and if statements. To illustrate we work with a basic subset of $\mathrm{C}$ and also use KLEE notation. Nevertheless, it should be noted that the proposed approach is generic and can be applied to many other language and symbolic execution tool combinations. Some annotations necessary to direct the transformation are identified using special comments "//\#" and given next:

high. The subsequent line has one or many secret variables. assume. The possible variables' values are limited by adding invariants. 
The first step is to partition the variables and make them symbolic. Only high variables are denoted. The step is illustrated in Program 1.2 .

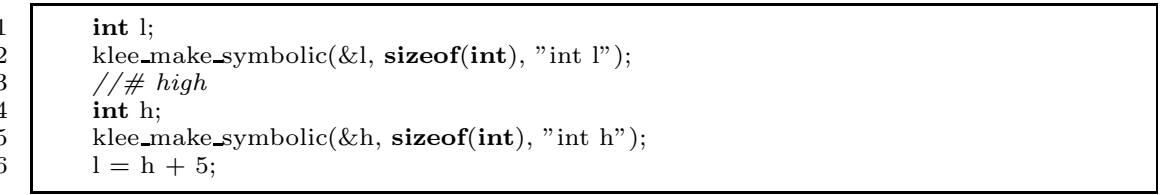

Program 1.2. Trivial noninterference example - labeled

The second step is to determine security types of expressions statically in the usual way: in essence, if an expression depends directly or indirectly on a high variable, it must be high.

The third step is to perform the necessary program transformations given in Figure 1, The rules used here are essentially Terauchi and Aiken's transformation [20] ported to a basic subset of C. The transformation is needed and used in the process of interleaving two copies of the candidate program. Note that the concrete rule applied for an if or while statement depends on whether the guard has high or low security type. If the guard is high the whole statements are composed sequentially, otherwise the bodies of the statements are interleaved.

$$
\begin{aligned}
& \frac{c \text { atomic }}{c \rightarrow c ; c^{\prime}} \quad \frac{c_{1} \rightarrow c_{1}^{\dagger} \quad c_{2} \rightarrow c_{2}^{\dagger}}{c_{1} ; c_{2} \rightarrow c_{1}^{\dagger} ; c_{2}^{\dagger}} \quad \frac{b \text { has low security type } c_{1} \rightarrow c_{1}^{\dagger} \quad c_{2} \rightarrow c_{2}^{\dagger}}{\text { if } b \text { then } c_{1} \text { else } c_{2} \rightarrow \text { if } b \text { then } c_{1}^{\dagger} \text { else } c_{2}^{\dagger}} \\
& \frac{b \text { has low security type } \quad c \rightarrow c^{\dagger}}{\text { while } b \text { do } c \rightarrow \text { while } b \text { do } c^{\dagger}} \quad \frac{b \text { has high security type }}{\text { while } b \text { do } c \rightarrow \text { while } b \text { do } c \text {; while } b^{\prime} \text { do } c^{\prime}} \\
& b \text { has high security type } \\
& \overline{\text { if } b \text { then } c_{1} \text { else } c_{2} \rightarrow \text { if } b \text { then } c_{1} \text { else } c_{2} ; \text { if } b^{\prime} \text { then } c_{1}^{\prime} \text { else } c_{2}^{\prime}}
\end{aligned}
$$

Fig. 1. Type-directed transformation [20]

The fourth and final stage of the transformation is to specify noninterference conditions. These are pre and post conditions derived from the program logic approach and guaranteeing that the program is secure. They assume that the low variables of the two copies are the same (and possibly some extra declassification conditions) and have to assert that the same holds at the end of the run. To illustrate the transformation approach, consider the annotated Program 1.3 .

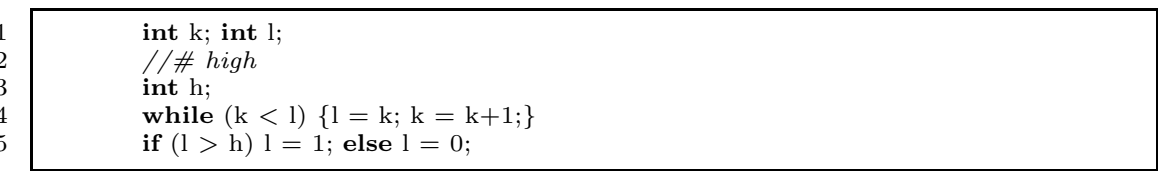

Program 1.3. Annotated program illustration

It is transformed into Program 1.4. 


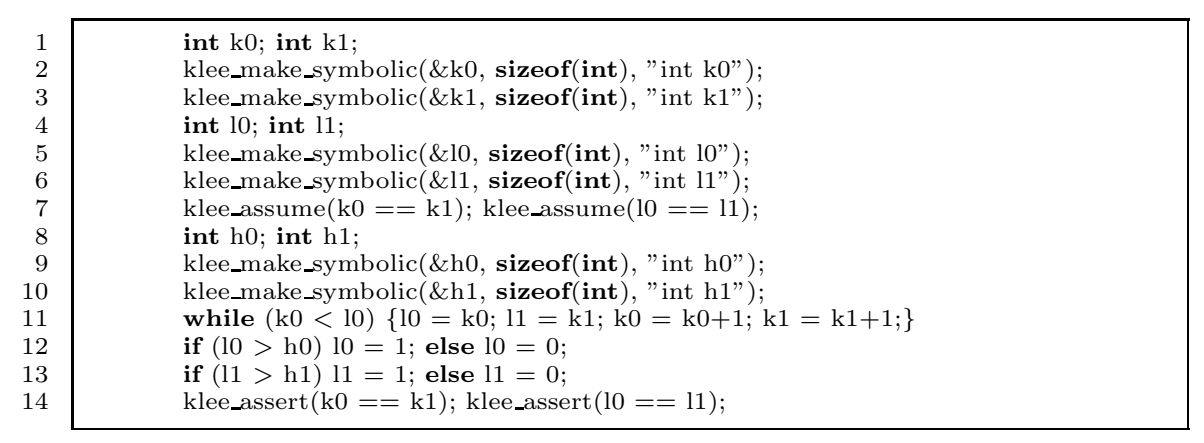

Program 1.4. Transformed program illustration

\subsection{Procedures}

Whereas Terauchi and Aiken develop their transformation for a very basic language, we need to deal with extra language features. One of these features is procedures: the rationale for transforming them is the same as for simple imperative programs. The transformation results in a new procedure with two copies of the parameters; if the original procedure has a non-void return type then two potentially different results of the same type are returned and thus have to be placed in a fresh struct.

In different cases variant(s) of the if and while rules from Fig. 1 have to be used. This depends on whether the procedure is called with arguments having high or low security types (or both) and is based on a respective data flow analysis. Consider Program 1.5 as an instance of a procedure to be transformed:

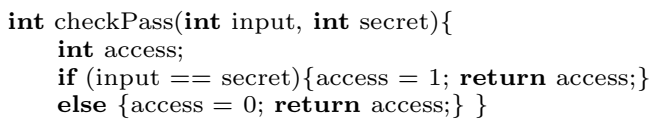

Program 1.5. Procedure with non-void return type

The transformed procedure should return a struct of two integers, but that means the original return statements have to be replaced with appropriate goto statements; these are used to make a transition to the second "copy" and ensure that a properly populated data structure is returned. The resulting transformation, assuming secret is passed a high value (thus second version of if rule used), is:

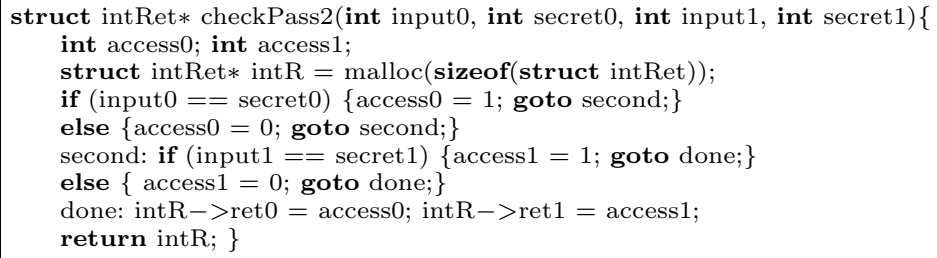

Program 1.6. Transformed procedure with non-void return type 
Next, we illustrate how to transform the int result $=$ checkPass (guess, pass $)$ procedure call (assuming the program consists of the call and variable assignments):

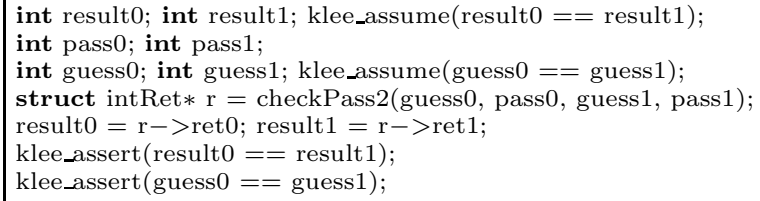

Note that $r$ is a "return" struct with two integer fields and variables have to be symbolic.

\subsection{Dynamically Allocated Data Structures and Noninterference}

It has already been suggested that different parts of a struct can be high or low. In order to model this and use symbolic execution to check programs allocating memory on the heap, we need to model the heap and change the noninterference definition respectively.

Let $F$ be a set of fields, $\mathcal{L}$ a set of locations and $\mathcal{V}=\mathbb{Z} \cup \mathcal{L} \cup\{$ null $\}$ be a set of values. A heap $\mathrm{h}$ will be modeled, following prior work [5], as a partial function $\mathrm{h}: \mathcal{L} \rightarrow S$; here $S=F \rightarrow \mathcal{V}$ is another partial function that models structs; the set of all heaps is Heap. Now $(\mathrm{m}, \mathrm{h}, P)$ denotes the final state after executing program $P$ with store $\mathrm{m}$ and heap $\mathrm{h}$. We write $(\mathrm{m}, \mathrm{h}, P)=\left(\mathrm{m}_{f}, \mathrm{~h}_{f}\right)$ to mean that the state evaluates to store $\mathrm{m}_{f}$ and heap $\mathrm{h}_{f}$. Let $\beta$ be a partial bijection on memory locations, used to model the low observer's uncertainty [2]. Let $v, v^{\prime} \in \mathcal{V}, L$ and $H$ be the low and high elements respectively of a typical security lattice. Value indistinguishability [5] can be defined as follows:

$$
v \sim_{\beta, H} v^{\prime} \quad \text { null } \sim_{\beta, L} \text { null } \quad \frac{v \in \mathbb{Z}}{v \sim_{\beta, L} v} \quad \frac{l, l^{\prime} \in \mathcal{L} \quad \beta(l)=l^{\prime}}{l \sim_{\beta, L} l^{\prime}} .
$$

Intuitively, two heaps $\mathrm{h}_{1}$ and $\mathrm{h}_{2}$ are indistinguishable if there is a bijection that relates each struct $s_{1}$ in heap $\mathrm{h}_{1}$ to its counterpart $s_{2}$ in heap $\mathrm{h}_{2}$; the structs have the same fields (because they are of the same type) and moreover the values of corresponding fields are indistinguishable. This is formally defined as follows: two heaps $\mathrm{h}_{1}, \mathrm{~h}_{2}$ are indistinguishable w.r.t. bijection $\beta$ denoted $\mathrm{h}_{1} \sim_{\beta} \mathrm{h}_{2}$ whenever: (1) $\operatorname{dom}(\beta) \subseteq \operatorname{dom}\left(\mathrm{h}_{1}\right)$ and $r n g(\beta) \subseteq \operatorname{dom}\left(\mathrm{h}_{2}\right)$; (2) for all $s \in \operatorname{dom}(\beta)$ we have that $\operatorname{dom}\left(\mathrm{h}_{1}(s)\right)=\operatorname{dom}\left(\mathrm{h}_{2}(\beta(s))\right.$ ) (for every struct in $\mathrm{h}_{1}$ its corresponding by $\beta$ struct in $\mathrm{h}_{2}$ has the same fields) and (3) for all fields $f \in \operatorname{dom}\left(\mathrm{h}_{1}(s)\right)$ with security level $L$ we have that $\mathrm{h}_{1}(s)(f) \sim_{\beta, L} \mathrm{~h}_{2}(\beta(s))(f)$, i.e. all field values of $\beta$-corresponding structs are $L$-indistinguishable. Similarly all fields with security level $H$ in corresponding structs have field values that are $H$-indistinguishable.

Definition 2. (Secure information flow [5]) A program $P$ is secure iff for all possible stores $m, m^{\prime} \in \operatorname{Var} \rightarrow \mathcal{V}$ and heaps $h, h^{\prime}, h_{f}, h_{f}^{\prime} \in$ Heap, and partial bijection $\beta$ such that $(m, h, P) \neq \perp$ and $\left(m^{\prime}, h^{\prime}, P\right) \neq \perp$, and $(m, h, P)=\left(m_{f}, h_{f}\right)$ and $\left(m^{\prime}, h^{\prime}, P\right)=\left(m_{f}^{\prime}, h_{f}^{\prime}\right)$, and $m \sim_{\beta} m^{\prime}$ and $h \sim_{\beta} h^{\prime}$ imply $m_{f} \sim_{\beta^{\prime}} m_{f}^{\prime}$ and $h_{f} \sim_{\beta^{\prime}, L}$ $h_{f}^{\prime}$ for some partial bijection $\beta^{\prime} \supseteq \beta$. 
The condition $\beta^{\prime} \supseteq \beta$ actually models the fact that new data structures may be dynamically created at runtime and thus the bijection may become larger. An illustration of the use of the new definition follows. The main function of a simplistic e-banking program is given in Program 1.7.

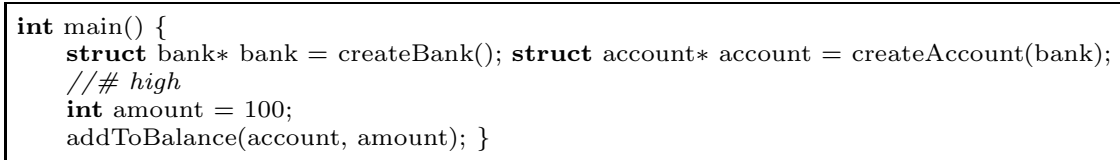

Program 1.7. Banking program - main

Each procedure call on line 2 declares and creates a struct. Each transformed procedure creates a pair of structs "packed" in another struct (see Section 3.3). The public fields of the struct are assumed equal. The result of the transformation is Program 1.8. The whole program, including procedure transformations, is available as Program 1.14 in Appendix A.

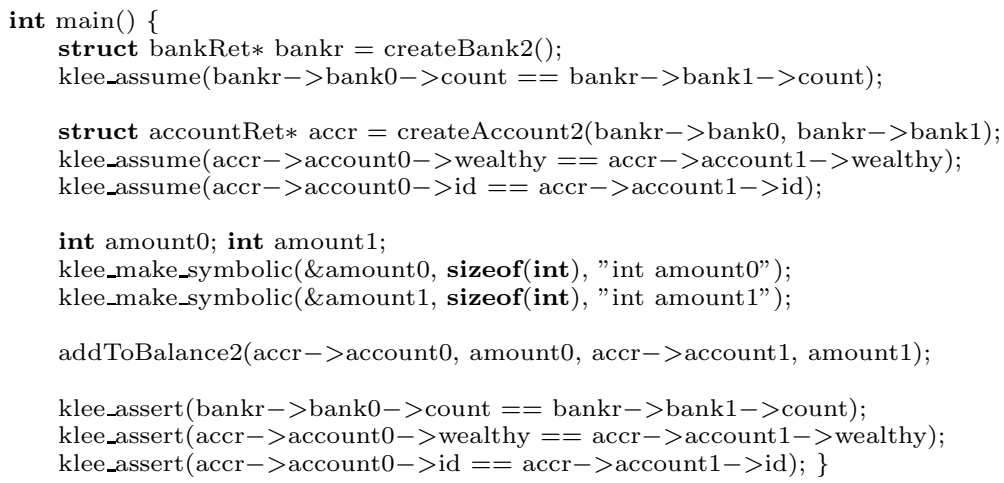

Program 1.8. Banking program - main transformation

In summary, whenever a new struct is allocated on the heap, the respective transformation allocates two structs and makes the appropriate assumptions about the low fields of the struct. At the end of execution, the respective assertions about the low structs have to hold. It should be noted that at this stage and particularly in the implementation of our tool, the bijection compares only the scalar values of the heap structure. The more general case, allowing cycles in the heap, is left for future work.

\section{Tool and Experimental Results}

This section presents a prototypical tool and some experimental results, demonstrating the potential of the proposed approach. 


\subsection{Tool Introduction}

The proof-of-concept tool that we have built to validate our ideas is written in Perl and is based on KLEE: an automatic symbolic execution tool for highcoverage test generation built on top of the LLVM compiler infrastructure 6]. The tool works with a subset of $\mathrm{C}$, including control flow statements and assignments to scalar variables, procedures and structs, where the fields have to be accessed in the standard way and no pointer arithmetic is allowed. Integer variables with addition and comparison operators are allowed as expressions.

Our tool takes as input a C program with specially annotated high variables, performs some program transformations, adds assertions as necessary and passes the resulting program to KLEE. Based on KLEE's output, the tool can possibly decide whether the tested program is secure or not and inform the developer or keep running indefinitely. In the latter case it cannot cover all paths, nevertheless it might still find a counterexample in the covered part and that would mean that the program is not secure. An optional parameter specifies when to time-out and stop searching. Whenever an error (assertion failure implying interference) is found, the ktest-tool tool (a part of the KLEE suite of tools) can be used to inspect and analyze the state that caused it. Additional data on the broken assertions is easily obtainable.

The tool can handle all the sample patterns of explicit and implicit information flow we could find in the literature. Furthermore it can handle patterns of information release. Because the transformations are based on semantic methods, the approach is more precise than information flow type systems resulting in the lack of false positives. On the other hand, the approach suffers from traditional weaknesses of symbolic execution, such as problems with scalability for large numbers of paths, dependence on the power of the constraint solver and difficult interaction with the environment. Moreover, the approach will benefit from further development of test input generation methods for programs with pointers. Despite the mentioned weaknesses, the tool is a very useful noninterference bug finder, as demonstrated in the rest of this section.

\subsection{Implicit Flow, Explicit Flow or No Flow}

Consider Program 1.9] it would be rejected as insecure by a typical information flow type system.

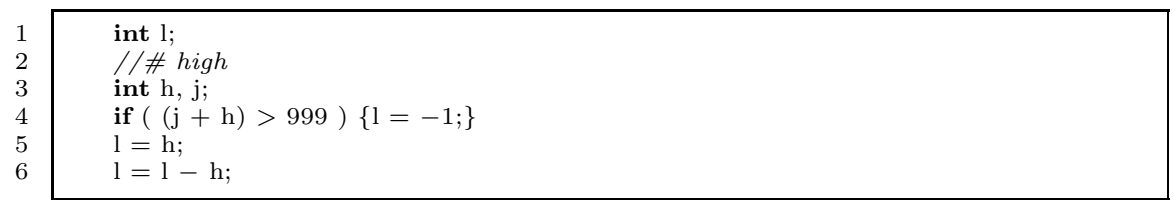

Program 1.9. Secure program rejected by flow-sensitive type systems

If we were to consider the program until and including the if statement, there would be an implicit flow; the program until and including the following 
statement $(l=h)$ would have both implicit and explicit flows. But Program 1.9 is secure: closer inspection shows that the leaks "neutralize" each other. The results are confirmed by our tool:

Program impexno.c secure.

\subsection{While-Loop Insecure Program [8]}

Consider Program 1.10, taken from prior work.

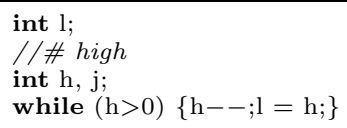

Program 1.10. While-loop insecure program

In order to prove the insecurity of this program, the theorem proving approach using the tool KeY takes 164 steps [8]; user interaction is needed for a number of steps, such as: establishing the induction hypothesis, instantiation and unwinding of the loop etc. Our tool detects the problem as expected and within $0.2 \mathrm{~s}$ (see Table 1 for detailed statistics):

Program while.c insecure. Flow in low variable l detected.

The tool is configured to stop after finding an error, but this is optional. More importantly, the developer has to only mark the high variable, which shields away the typical complexity imposed by alternative approaches (e.g. verification ones). The developer does not need to be a verification expert or to think about loopinvariants, unwinding, assertions, etc. but nevertheless has a powerful testing tool at her disposal.

\section{4 e-Banking Example}

The e-banking program of Section 3.4 is presented next. The interesting, securityrelated code is in procedure addToBalance2:

if (amount $>=10000)$ account $->$ wealthy $=$ true; else account $->$ wealthy $=$ false;

Program 1.11. Security-related part of e-Banking example

Whenever the balance gets higher than 10000, flag wealthy is set. The field wealthy of the struct account is public and leaks information about the balance. The latter is confirmed by our tool, producing the following output: 


\subsection{Average Example}

Recall that Program 1.1 computes the average of two high variables. Preconditions on the values of variables, such as //\#assume $(i>0 \& j>0)$, can be specified. As already discussed, the program is not secure and the tool terminates with the appropriate error:

\section{Program avg.c insecure. Flow in low variable $l$.}

It should be noted that our tool is precise in the sense that the errors are reproducible. The values that broke the assertions can be inspected using KLEE's ktest-tool in order to analyze the problem. The values generated by the ktest-tool are: $l 0=0, l 1=0, i 0=506, i 1=1609415267, j 0=507, j 1=485081005$.

\subsection{Password Examples}

Next consider the simple password check in Program 1.12

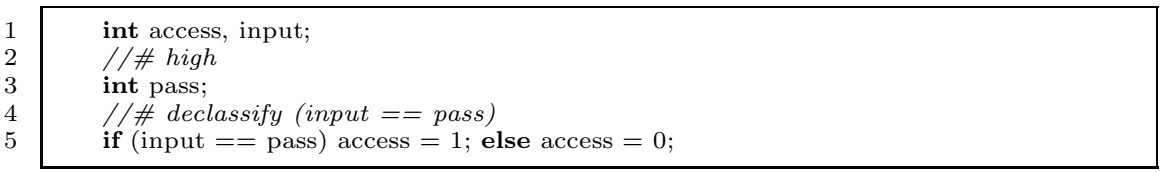

\section{Program 1.12. Password check}

Password checking programs leak information as a part of their functionality. This can be seen if we consider the program without line 4; even when a given guess is wrong, guessing reveals that the password is or is not equal to the guess. This trivial leak is detected as expected:

Program passw.c insecure. Flow in low variable access.

As a result of the declassify statement though, the extra condition $(($ input0 $==$ pass 0$)==($ input $1==$ pass 1$))$ is added to the the assumptions; this is of course transparent to developers. In this case, our tool verifies that Program 4.6 is secure:

Program passwDecl.c secure.

Finally, we consider the following program, illustrating the use of procedures:

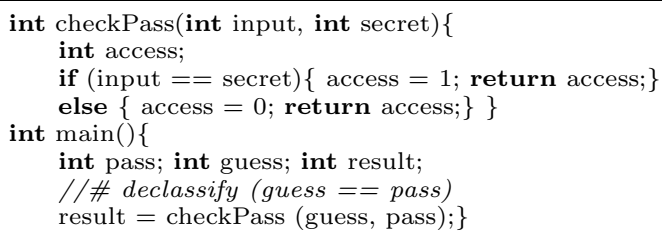

Program 1.13. Password example with procedures

The program is secure, as expected. 


\section{7 $\quad$ Statistics}

Statistics of the discussed examples are presented in Table 1, Each of the considered programs is characterized by the respective number of instructions, explored paths and generated test cases (given by KLEE). Finally the output of the time program is given; real time is the elapsed time between start and finish, whereas user gives the CPU time spent in user mode and sys gives the CPU time in kernel mode. Typically user and sys time tell us how much CPU time the process used. The highest time by this criterion is below $0.25 \mathrm{~s}$. It should be noted that the presented examples include ones whose verification would require a considerable effort if a special purpose type system would have to be developed (for each slightly different definition of security) or a theorem prover would have to be used. Because instruction cycles are cheap nowadays, a tool based on the proposed approach has a high potential of being very useful in everyday development work.

The considered examples are not particularly large, but we have tried embedding them deeper in realistic programs and the results appear to be promising. It should be noted that the definitions considered here (and in the larger part of the security literature) are of termination insensitive notions of security.

Table 1. Statistics for presented programs

\begin{tabular}{|c|c|c|c|c|c|c|}
\hline Program & Instructions & Paths & Generated Tests & \multicolumn{3}{|c|}{ Time } \\
\cline { 4 - 6 } & & & & real & user & sys \\
\hline 1.9 & 118 & 4 & 4 & $0.199 \mathrm{~s}$ & $0.062 \mathrm{~s}$ & $0.023 \mathrm{~s}$ \\
\hline 1.10 & 57 & 5 & 5 & $0.134 \mathrm{~s}$ & $0.050 \mathrm{~s}$ & $0.024 \mathrm{~s}$ \\
\hline 1.7 & 430 & 4 & 3 & $0.095 \mathrm{~s}$ & $0.046 \mathrm{~s}$ & $0.024 \mathrm{~s}$ \\
\hline 1.1 & 156 & 4 & 3 & $0.268 \mathrm{~s}$ & $0.212 \mathrm{~s}$ & $0.030 \mathrm{~s}$ \\
\hline 1.12 (insecure) & 76 & 4 & 3 & $0.099 \mathrm{~s}$ & $0.053 \mathrm{~s}$ & $0.024 \mathrm{~s}$ \\
\hline 1.12 (secure) & 76 & 2 & 2 & $0.089 \mathrm{~s}$ & $0.051 \mathrm{~s}$ & $0.021 \mathrm{~s}$ \\
\hline 1.13 & 153 & 2 & 2 & $0.108 \mathrm{~s}$ & $0.055 \mathrm{~s}$ & $0.025 \mathrm{~s}$ \\
\hline
\end{tabular}

\section{Related Work}

To the best of our knowledge, we are the first to propose the use of symbolic execution for testing noninterference, boasting the advantages of precision and full automation not available in typical verification approaches. Nevertheless, many of the ideas presented here appear in the substantial literature on verification of noninterference. Proposed solutions traditionally rely on information flow type systems [16], a syntactic approach which offers an overapproximation and thus tends to be too conservative in practice; moreover, a new type system has to be developed every time a slight modification of the needed notion of security is needed (e.g. to allow a specific notion of declassification). On the other hand, many attempts to address noninterference have a semantic flavor 912 ; such approaches are attractive because they suggest methods to transform the problem so as to benefit from state-of-the-art verification techniques and tools. These 
approaches gave rise to further work on program-logics based characterizations of noninterference [84: both these rely on the idea of reducing noninterference of a program to a property of the sequential composition of the program with itself. Reasoning about such constructs is facilitated by Terauchi and Aiken's type-directed transformation [20], which takes advantage of the structure of a self-composed program and the resulting symmetry and redundancy.

In the most relevant related work Backes et al. 1] use techniques similar to ours to compute all information leaks in a program and to quantify the leaks using information-theoretic means. Information leaks in their work are characterized by an equivalence relation on secrets and can be expressed as a logical assertion on program variables; this is similar to our approach. They start with a relation expressing noninterference and gradually refine it, when counterexamples are found. Their quantitative analysis is based on computing the number and sizes of equivalence classes. The proposed approach computes an overapproximation of the information leaked by a program (and then checks if such a relation is reachable from the start state) unlike our approach, based on a finer relation (we rely on an underapproximation, only reachable states are considered). Another related and important difference is that our approach does not require a set of experiments to start with, due to the nature of symbolic execution. This is an advantage because it makes the approach more automatic. There are also differences between the approaches on other levels. First, we address a slightly different problem: testing a program for conformance with a base-line information flow policy, possibly augmented with a notion of information release, giving direct feedback to developers and being fully automatic; second, we use symbolic execution, whereas their approach uses off-the-shelf model checkers; finally, we explore qualitative policies only.

Declassification is also a well-studied topic (see [18 for an overview). The idea to use equivalence relations to characterize partial information flow was originally proposed by Cohen [7] and further developed in the literature [2310]. A number of related articles explore the use of equivalence relations to characterize information release using flow-sensitive type systems [1417. Declassification is similarly handled in work using the KeY tool 8, but again the difference is that symbolic execution there is used for verification.

\section{Conclusion}

We have presented a novel, automatic approach to testing noninterference: the only responsibility of the developer is to identify the secrets in a candidate program and appropriately annotate them. The program is then automatically transformed and assertions are added as needed. Next, dynamic symbolic execution is used to try to break the assertions. Because typically bugs are shallow, the approach has a high potential to be very useful for testing; a major advantage of the approach is precision: any assert violation indicates a concrete, reproducible security bug. Moreover, there is no need to write complex invariants or be an expert in verification to assist the tool in any way and this can be seen as a major advantage for developers. 
To illustrate the usefulness of the proposed approach, we have built a prototypical tool based on the symbolic execution tool KLEE. Our tool takes as input an annotated program from a "well-behaved" subset of C, performs the necessary program transformations and passes the resulting program to KLEE. Then, based on KLEE's output the tool informs whether a bug could be found. We have successfully tested the tool on a number of programs known from the literature and exhibiting patterns of insecurity.

Acknowledgements. We would like to thank Dries Vanoverberghe for very insightful and valuable comments on a late draft of the paper and Tatyana Doktorova for many helpful suggestions on the presentation. We also thank the reviewers for their constructive feedback.

\section{References}

1. Backes, M., Kopf, B., Rybalchenko, A.: Automatic discovery and quantification of information leaks. In: Proceedings of the 2009 30th IEEE Symposium on Security and Privacy, pp. 141-153. IEEE Computer Society, Washington, DC (2009)

2. Banerjee, A., Naumann, D.A.: Stack-based access control and secure information flow. Journal of Functional Programming 15, 131-177 (2005)

3. Banerjee, A., Naumann, D.A., Rosenberg, S.: Towards a logical account of declassification. In: Proceedings of the 2007 Workshop on Programming Languages and Analysis for Security, PLAS 2007, pp. 61-66. ACM, New York (2007)

4. Barthe, G., D'Argenio, P.R., Rezk, T.: Secure information flow by self-composition. In: Proceedings of the 17th IEEE workshop on Computer Security Foundations, pp. 100-114. IEEE Computer Society, Washington, DC (2004)

5. Barthe, G., Rezk, T.: Non-interference for a JVM-like language. In: TLDI 2005, pp. 103-112. ACM, New York (2005)

6. Cadar, C., Dunbar, D., Engler, D.: KLEE: unassisted and automatic generation of high-coverage tests for complex systems programs. In: OSDI 2008, pp. 209-224. USENIX Association, Berkeley, CA (2008)

7. Cohen, E.S.: Information transmission in sequential programs. In: DeMillo, R.A., Dobkin, D.P., Jones, A.K., Lipton, R.J. (eds.) Foundations of Secure Computation, pp. 297-335. Academic Press (1978)

8. Darvas, Á., Hähnle, R., Sands, D.: A theorem proving approach to analysis of secure information flow. Technical Report S-412 96, Chalmers University of Technology and Göteborg University (2004)

9. Focardi, R., Gorrieri, R.: A taxonomy of security properties for process algebras. Journal of Computer Security 3(1), 5-34 (1995)

10. Giacobazzi, R., Mastroeni, I.: Abstract non-interference: Parameterizing noninterference by abstract interpretation. In: Proc. of the 31st Annual ACM SIGPLAN-SIGACT Symposium on Principles of Programming Languages (POPL 2004), pp. 186-197. ACM Press, NY (2004)

11. Godefroid, P., Klarlund, N., Sen, K.: Dart: directed automated random testing. In: Proceedings of the 2005 ACM SIGPLAN Conference on Programming Language Design and Implementation, PLDI 2005, pp. 213-223. ACM, New York (2005)

12. Joshi, R., Leino, K.R.M.: A semantic approach to secure information flow. Science of Computer Programming 37, 113-138 (2000) 
13. King, J.C.: Symbolic execution and program testing. Communications of the ACM 19, 385-394 (1976)

14. Myers, A.C., Sabelfeld, A., Zdancewic, S.: Enforcing robust declassification and qualified robustness. Journal of Computer Security 14(2), 157-196 (2006)

15. Pottier, F., Simonet, V.: Information flow inference for ML. SIGPLAN Not. 37, 319-330 (2002)

16. Sabelfeld, A., Myers, A.C.: Language-based information-flow security. IEEE Journal on Selected Areas in Communications 21(1), 5-19 (2003)

17. Sabelfeld, A., Myers, A.C.: A Model for Delimited Information Release. In: Futatsugi, K., Mizoguchi, F., Yonezaki, N. (eds.) ISSS 2003. LNCS, vol. 3233, pp. 174-191. Springer, Heidelberg (2004)

18. Sabelfeld, A., Sands, D.: Dimensions and principles of declassification. In: Proceedings of the 18th IEEE Workshop on Computer Security Foundations, pp. 255-269. IEEE Computer Society, Washington, DC (2005)

19. Sen, K., Marinov, D., Agha, G.: CUTE: a concolic unit testing engine for C. SIGSOFT Software Engineering Notes 30, 263-272 (2005)

20. Terauchi, T., Aiken, A.: Secure Information Flow as a Safety Problem. In: Hankin, C., Siveroni, I. (eds.) SAS 2005. LNCS, vol. 3672, pp. 352-367. Springer, Heidelberg (2005)

21. Volpano, D.M., Smith, G.: A Type-Based Approach to Program Security. In: Bidoit, M., Dauchet, M. (eds.) CAAP/FASE/TAPSOFT 1997. LNCS, vol. 1214, pp. 607-621. Springer, Heidelberg (1997)

22. Zdancewic, S., Myers, A.C.: Observational determinism for concurrent program security. In: Proceedings 16th IEEE Computer Security Foundations Workshop, pp. 29-43 (July 2003)

23. Zdancewic, S., Myers, A.C.: Robust declassification. In: Proceedings of the 14th IEEE Workshop on Computer Security Foundations, CSFW 2001, pp. 15-23. IEEE Computer Society, Washington, DC (2001) 


\section{A Sample Transformation}

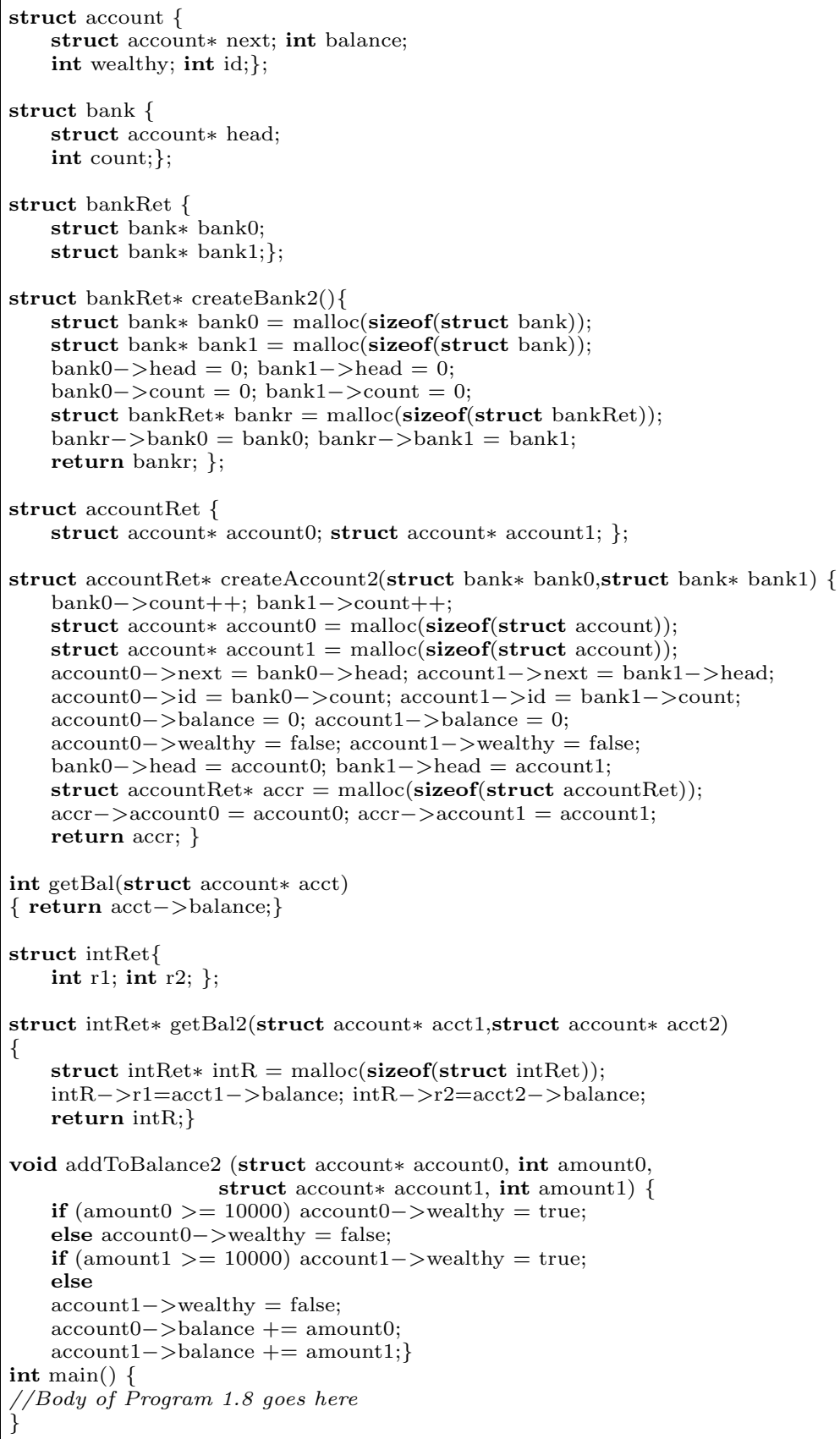

Program 1.14. Transformation and noninterference specification of Program 1.7 